Check for updates

Cite this: Mater. Adv., 2022, 3, 946

Received 29th September 2021 Accepted 26th November 2021

DOI: $10.1039 / \mathrm{d} 1 \mathrm{ma} 00897 \mathrm{~h}$

rsc.li/materials-advances

\section{Investigation of a 2D WS 2 nanosheet-reinforced tough DNA hydrogel as a biomedical scaffold: preparation and in vitro characterization $\uparrow$}

\author{
Sayantani Basu, ${ }^{a}$ Aishik Chakraborty, $\ddagger^{\mathrm{b}}$ Abdul-Rahman Imad Alkiswani, $\ddagger^{\mathrm{c}}$ \\ Yasmeen Shamiya ${ }^{d}$ and Arghya Paul (D) *e
}

Hydrogels, prepared from natural polymers, are attractive biomaterials for diverse biomedical applications due to their excellent biocompatibility and bioactivity. However, the majority of conventional hydrogels are mechanically weak and unsuitable for use in the repair of load-bearing tissues. Herein, we utilized DNA as a natural biopolymer and two-dimensional nanosheets of tungsten disulfide $\left(\mathrm{WS}_{2}\right)$ to engineer mechanically tough nanocomposite hydrogels. Single network hydrogels were formed via covalent crosslinking of DNA chains in the presence of alginate-exfoliated $\mathrm{WS}_{2}$ nanosheets. A polyethylene glycol-based bi-functional crosslinker with epoxide end groups was used to join the DNA strands via chemical crosslinking. Thereafter, the alginate chains in the hydrogel formulation were ionically crosslinked with $\mathrm{Ca}^{2+}$ ions to form double network polymeric hydrogels. Oscillatory shear rheology and uniaxial compression testing elucidated the beneficial effects of nanosheets and the formation of double network on the mechanical and structural properties of resulting hydrogels. Significant enhancement in compressive moduli and yield stress further corroborated the reinforcing effects of $\mathrm{WS}_{2}$ and secondary network. Improved mechanical properties increase the applicability of these nanocomposites because of their enhanced tissue-mimicking abilities. In vitro cytotoxicity assays with human stem cells confirmed the biocompatibility of formulated nanocomposite hydrogels. To conclude, we envision these tough DNA-based hydrogels can be effectively used in the future as a scaffold for various biomedical applications, including delivery of drugs such as proteins, growth factors, small molecules, among others.

\section{Introduction}

In recent years, two-dimensional (2D) transition metal dichalcogenides (TMDs) such as molybdenum disulfide $\left(\mathrm{MoS}_{2}\right)$ and tungsten disulfide $\left(\mathrm{WS}_{2}\right)$ have shown immense potential to be used as a new class of materials in a diverse range of biomedical applications, including controlled drug delivery, biosensing, and formation of tissue engineering scaffolds, among others. ${ }^{1-3}$ This applicability stems from their high surface area and distinctive mechanical, structural, and antimicrobial properties. ${ }^{4-7}$ Moreover, 2D TMDs are excellent photothermal agents that can convert near-infrared light into thermal energy. ${ }^{8,9}$ As a result, 2D TMDs are gaining popularity as vehicles for contact-free lighttriggered drug delivery and as photothermal agents for the ablation of tumors. ${ }^{10-12}$ Despite their enormous potential, a major limiting factor for the use of TMDs in biomedical applications is their water insolubility and hence difficulty to exfoliate in aqueous media, a process that ultimately leads to the formation of ultrathin sheets. However, few recent studies have exploited the possibility of using bovine serum albumin, sodium alginate, as well as DNA strands as exfoliating agents. ${ }^{13-16}$ Recently, a polyvinyl pyrrolidone exfoliated 2D tungsten disulfide nanosheet was used for in vivo thermal imaging and treating colon adenocarcinoma. ${ }^{17}$ Such exfoliated, ultrathin 2D nanosheets of TMDs have been incorporated within hydrogel-based therapeutic devices for biomedical applications. $^{18,19}$

Hydrogels are three-dimensional (3D), hydrophilic, crosslinked polymeric network-based biomaterials that can retain

\footnotetext{
${ }^{a}$ Department of Chemical and Petroleum Engineering, School of Engineering, University of Kansas, Lawrence, Kansas 66045, United States Department of Biomedical Engineering, University of Rochester, Rochester, New York 14627, USA

${ }^{b}$ Department of Chemical and Biochemical Engineering, The University of Western Ontario, London, Ontario N6A 5B9, Canada

${ }^{c}$ School of Medicine, University of Kansas Medical Centre, Kansas, Kansas 66160, USA

${ }^{d}$ Department of Chemistry, The University of Western Ontario, London, ON N6A 5B9, Canada

${ }^{e}$ Department of Chemical and Biochemical Engineering, Department of Chemistry, School of Biomedical Engineering, The Centre for Advanced Materials and Biomaterials Research, The University of Western Ontario, London, ON N6A 5B9, Canada. E-mail: arghya.paul@uwo.ca

$\dagger$ Electronic supplementary information (ESI) available. See DOI: 10.1039/d1ma00897h

\$ Equal contributions.
} 
large quantities of water, making them appealing for both drug delivery and tissue engineering applications. ${ }^{20-25}$ However, traditional hydrogels often lack in terms of their responsiveness to chemical ( $\mathrm{pH}$, ionic strength) or physical (light, magnetic field) stimuli or may display weak physical properties. ${ }^{26}$ To improve the functionality and the applicability of hydrogels, nanomaterials are often incorporated within these crosslinked polymeric networks, forming external stimuli-responsive, mechanically robust nanocomposite hydrogels. ${ }^{27-30} 2 \mathrm{D}$ TMDs have been used to enhance the mechanical properties of hydrogels, thereby increasing the applicability of natural polymers. $^{31}$ DNA can be one such natural, generic, preciselyprogrammable polymer that can be paired with nanomaterials to synthesize advanced nanocomposite hydrogels for biomedical applications. $^{32-37} \mathrm{Xu}$ et al. fabricated a self-assembled, multifunctional hydrogel containing DNA and graphene oxide. ${ }^{38}$ Recently, Pandey et al. developed a TMD quantum dot reinforced fluorescent DNA hydrogel. ${ }^{39}$ When paired with 2D TMDs, such multifunctional, biocompatible nanocomposite hydrogels can show enhanced responsiveness to near-infrared light stimuli and favorable physical properties, such as porous network structures for cell infiltration.

Herein, we have designed one such DNA-based hydrogel platform, reinforced with $2 \mathrm{D}$ nanosheets of $\mathrm{WS}_{2}\left(\mathrm{WS}_{2}\right.$-Alg) to form a nanocomposite scaffold suitable for biomedical applications. We have exfoliated $\mathrm{WS}_{2}$ powder into ultrathin nanosheets by dispersing the powder in an aqueous solution of sodium alginate (Na-Alg), followed by vigorous sonication. The exfoliated $\mathrm{WS}_{2}$-Alg nanosheets were then used to reinforce a covalently crosslinked single network DNA hydrogel. Finally, the alginate used for the exfoliation of $\mathrm{WS}_{2}-\mathrm{Alg}$ nanosheets was crosslinked by the addition of calcium chloride to generate a mechanically robust double network DNA based hydrogel. Such double network hydrogels contain a combination of interconnected polymer networks and hence demonstrate significantly improved mechanical properties. ${ }^{40,41}$ The formulated hydrogels

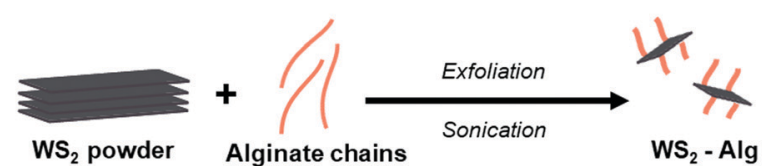

D TEM micrograph of $\mathrm{WS}_{2}-\mathrm{Alg}$

B Comparing WS $_{2}$ - Alg with the precursor material
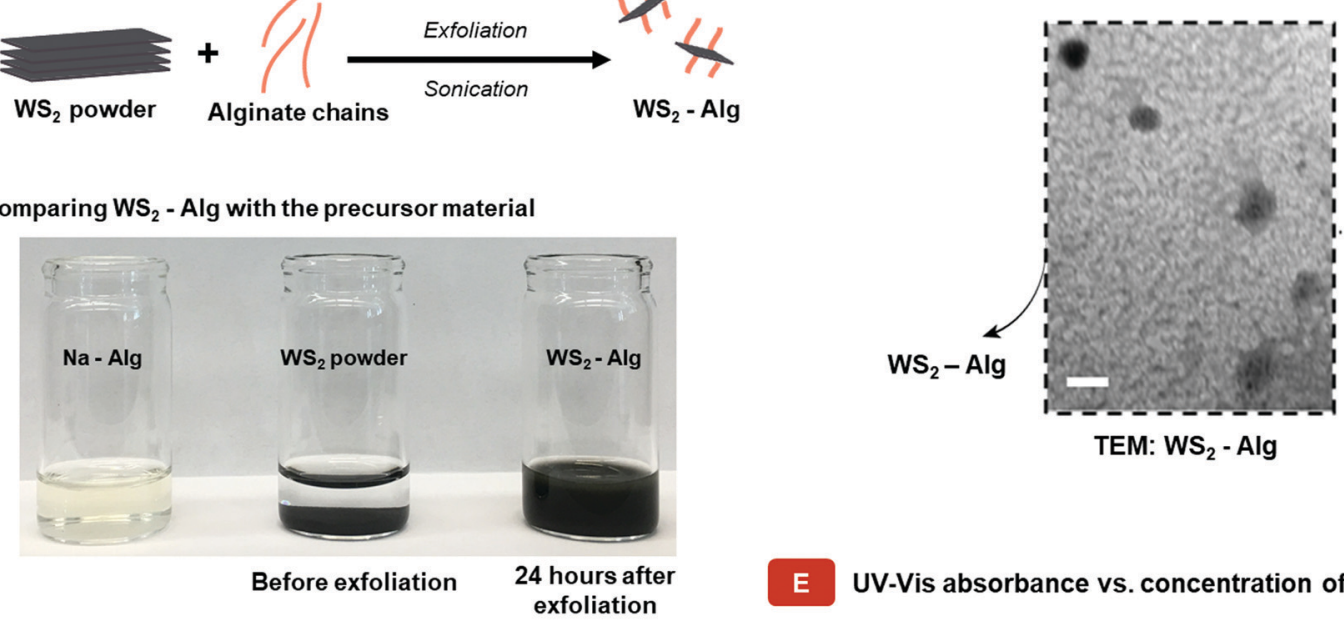

TEM: WS $_{2}-$ Alg

E UV-Vis absorbance vs. concentration of $\mathrm{WS}_{2}-\mathrm{Alg}$

Time-resolved stability of $\mathrm{WS}_{2}$ - Alg vs. concentration

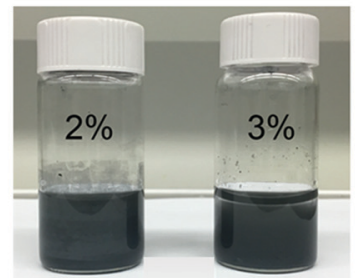

Day 2

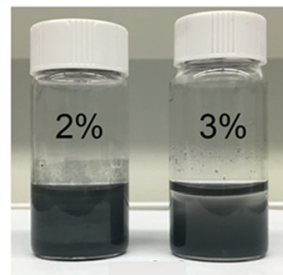

Day 3

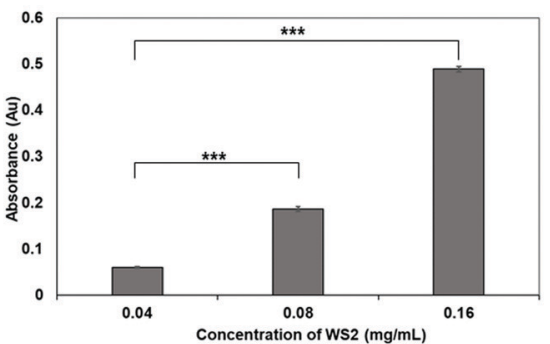

Fig. 1 Synthesis and appearance of two-dimensional $W_{2}$-Alg nanosheets. (A) Schematic showing the process of preparing ultrathin, two-dimensional nanosheets of tungsten disulfide $\left(\mathrm{WS}_{2}\right.$ ) using tungsten disulfide powder and sodium alginate (Na-Alg) solution. WS 2 powder was suspended in a $1 \%$ ( $\mathrm{W} / \mathrm{v}$ ) sodium alginate solution and then sonicated for exfoliation into nanosheets. (B) Pictures comparing the sonicated precursor solutions of $1 \%$ sodium alginate and $1 \%$ tungsten disulfide with exfoliated, two-dimensional $1 \%$ tungsten disulfide. After 24 hours, the sonicated WS 2 powder precursor sedimented, whereas the $\mathrm{WS}_{2}$-Alg nanosheets stayed stable in suspension. (C) Images showing the appearance of $2 \%$ and $3 \%$ exfoliated WS 2 -Alg after 2 and 3 days. $3 \% \mathrm{WS}_{2}$-Alg displayed aggregation and settled down with minimum stability over extended time spans, whereas $2 \% \mathrm{WS}_{2}$-Alg remained stable after 3 days. (D) Transmission electron micrograph displaying the morphology of ultrathin, two-dimensional nanosheets of $2 \% \mathrm{WS}_{2}$-Alg (scale bar $=$ $50 \mathrm{~nm}$ ). (E) Graph displaying the increase in UV-vis absorbance (at $630 \mathrm{~nm}$ wavelength) with an increase in the concentration of exfoliated WS 2 -Alg nanosheets. Results are represented as mean \pm standard deviation $(n=3)(* * * p 0.001)$. 
were characterized via dynamic mechanical analysis and oscillatory shear rheology to illustrate the effect of both $\mathrm{WS}_{2}$-Alg nanosheets and the second network of ionically crosslinked alginate chains on the mechanical properties of the DNA-based platform. Next, the physical properties, i.e. the morphology and the swelling behavior, of the hydrogels were monitored. Physical characterization was followed by examining the biocompatibility of the developed nanocomposite hydrogels by in vitro cytotoxicity assays with human adipose stem cells (hASCs). Lastly, we propose a variety of biomedical applications of the designed DNA-based hydrogel.

\section{Results and discussion}

\subsection{Preparation of exfoliated ultrathin 2D nanosheets of tungsten disulfide}

Fig. 1A illustrates the method for exfoliating 2D $\mathrm{WS}_{2}$-Alg nanosheets. Polymers are widely used for exfoliating $\mathrm{WS}_{2}$ powder to form nanosheets. ${ }^{16,42}$ Following literature, we used $1 \%$ sodium alginate as the exfoliating solution. ${ }^{13} \mathrm{WS}_{2}$ belongs to the class of transition metal dichalcogenides, which makes it structurally similar to $\mathrm{MoS}_{2}$. Fig. 1B shows the appearance of exfoliated and sonicated $\mathrm{WS}_{2}$-Alg nanosheets after 24 hours of exfoliation. Fig. 1C demonstrates the stability of $\mathrm{WS}_{2}-\mathrm{Alg}$ as a function of concentration. It was observed that the one with $2 \%$ nanosheets remain dispersed in suspension even at 3 days after exfoliation. However, 3\% nanosheets aggregate and sediment within 3 days. Next, we observed irregular lateral morphology of the exfoliated nanosheets using transmission electron microscopy (Fig. 1D). Similar morphology has been reported elsewhere. ${ }^{16}$ Fig. 1E displays the concentration-dependent increase in ultraviolet-visible (UV-vis) absorption at $630 \mathrm{~nm}$, which is characteristic of the $\mathrm{WS}_{2}$-Alg nanosheets. ${ }^{43}$

\subsection{Fabrication and mechanical characterization of single network nanocomposite DNA hydrogels}

Fig. 2A displays the method for synthesizing the tungsten disulfide-impregnated single network DNA hydrogel. The formulated hydrogels with $0.1875 \%, 0.375 \%$, and $0.75 \% \mathrm{WS}_{2}$ concentrations (w/v\%) are labelled as "Low", "Medium", and "High", respectively. The hydrogel without any $\mathrm{WS}_{2}$ is designated as "Gel". The structural and mechanical properties of the designed single network DNA hydrogel reinforced with the three different concentrations of $\mathrm{WS}_{2}$-Alg were determined by uniaxial compression testing. The stress vs. strain plots displayed an increase in the ultimate stress with the addition of exfoliated $\mathrm{WS}_{2}$ to the DNA-based system (Fig. 2B). The compressive moduli were calculated from the slope of the stress $v s$. strain curves in the initial region of $0.1-0.2 \mathrm{~mm} \mathrm{~mm}^{-1}$ strain. A significant increase in the compressive modulus from $3.62 \mathrm{kPa}$ to $8.13 \mathrm{kPa}$ was detected with an increase in $\mathrm{WS}_{2}$ concentration from 0 to $0.75 \%$. Previous studies have reported the use of $\mathrm{WS}_{2}$ for the formation of reinforced composites in conjunction with biodegradable polyesters and poly(L-lactic acid) for bone tissue engineering. ${ }^{44,45}$ Although not studied here, the large surface area and high aspect ratio of these twodimensional nanosheets make them ideal candidates as nanofillers for the development of tissue engineering scaffolds. ${ }^{46}$ Next, we monitored the behavior of the developed DNA-WS

Chemical method to synthesize WS $_{2}$-impregnated single-network DNA-based hydrogel
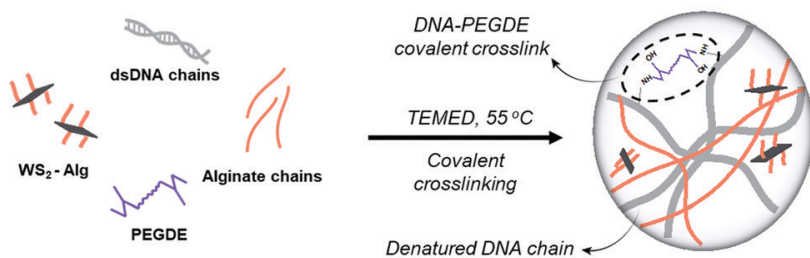

Mechanical characterization of DNA-based hydrogel with varying concentrations of $\mathrm{WS}_{2}$
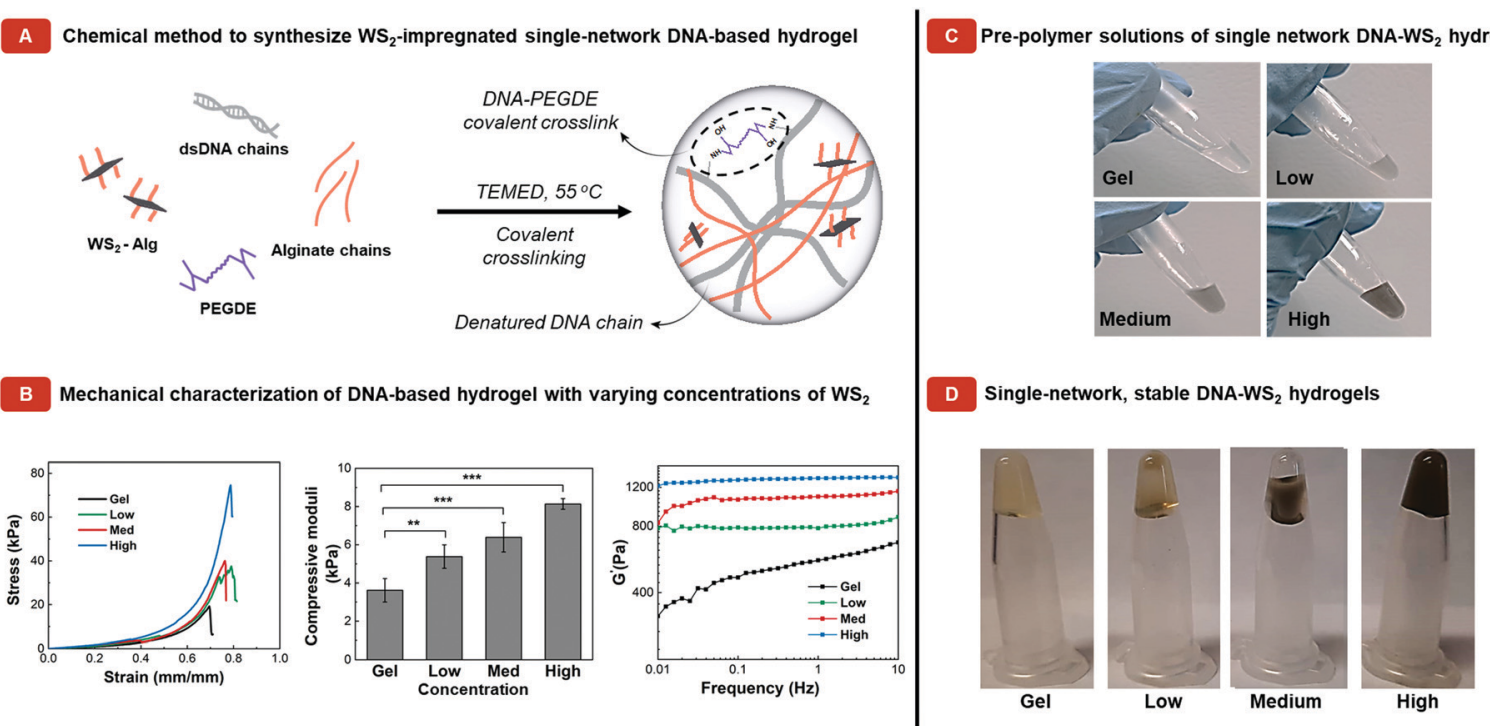

Fig. 2 Strategy for fabricating single network DNA-WS 2 hydrogels and characterizing their mechanical behavior. (A) Schematic depicting the method for developing single network DNA-WS 2 hydrogels. Here, double stranded DNA (dsDNA) chains were covalently crosslinked with polyethylene glycol diepoxide (PEGDE) to form the desired nanoparticle-impregnated material. (B) Stress vs. strain plots of the DNA-WS 2 hydrogels containing different concentrations ("Low", "Medium", and "High") of WS 2 . "Gel" represents the hydrogel without nanoparticles. Comparison of the compressive moduli of the DNA-WS 2 gels containing different concentrations of $W_{2}$. Results are represented as mean \pm standard deviation $(n=4)\left({ }^{* * *} p<0.001\right)$. Frequencysweep results of the DNA-WS ${ }_{2}$ gels containing different concentrations of $W_{2}$. (C) Images showing the appearance of precursor solutions of single network DNA-WS 2 hydrogels impregnated with different concentrations of WS 2 nanosheets. (D) Pictures depicting the same formulations after gelation. The stable nanocomposite hydrogels remain in place even after inverting the microcentrifuge vials. 
hydrogel under the application of shear forces via oscillatory shear rheology. The storage modulus values of the "High" gel were observed to be the highest over the entire range of tested frequencies. This improved mechanical performance can be attributed to the presence of non-covalent interactions between the surface of the dichalcogenide and nitrogenous bases of DNA strands ${ }^{[10]}$. Previous studies have demonstrated the presence of Van der Waals interactions between DNA and $\mathrm{WS}_{2}$-Alg nanosheets. ${ }^{4,48}$ The presence of these interactions results in the formation of reinforced nanocomposites with enhanced compressive and storage moduli as well as higher yield stress values. Fig. 2C and D displays the prepolymer solutions and the corresponding gels that were tested here. However, the formulation designated as "High" was selected for further studies since it provided the best set of mechanical properties.

\subsection{Development and mechanical characterization of double network DNA-WS ${ }_{2}$ hydrogels}

Following the optimization of the nanosheet concentration in the single network DNA hydrogel based on its mechanical properties, we crosslinked the alginate chains with $\mathrm{Ca}^{2+}$ to formulate the double network structure. Fig. 3A shows the process by which the designed single network DNA hydrogel was converted to a double network hydrogel. Alginate aqueous solution $(1 \% \mathrm{w} / \mathrm{v})$ was used to exfoliate $\mathrm{WS}_{2}$-Alg into nanosheets for the exploitation of their maximum potential as reinforcing nanofillers. Sodium alginate has been previously used for its capability to intercalate between the dichalcogenide layers for achieving extensive exfoliation. ${ }^{13}$ An aqueous solution of sodium alginate has been successfully employed for the exfoliation of $\mathrm{MoS}_{2}$ which possesses similar structural properties to $\mathrm{WS}_{2} \cdot{ }^{13}$ For this reason, we used alginate as the dispersing agent in our studies for fabricating the DNA nanocomposites. Consequently, the DNA- $\mathrm{WS}_{2}$ hydrogels contained alginate chains distributed in the single network of covalently crosslinked DNA chains. The double network hydrogels were formed by immersing the $\mathrm{DNA}^{-\mathrm{WS}_{2}}$ nanocomposite hydrogels in $0.025 \mathrm{M}$ calcium chloride aqueous solutions. Divalent $\mathrm{Ca}^{2+}$ ions interact with the carboxylate groups mainly on the guluronate residues of the alginate backbone, creating an ionically crosslinked alginate network. ${ }^{49}$

Next, we investigated the effect of adding the second network on the structural and mechanical properties of the formulated hydrogels. The single network hydrogels were designated as SN-High, where High denotes the highest concentration $(0.75 \%)$ of $\mathrm{WS}_{2}$-Alg. The double network hydrogels were labelled as DN-High. The stress vs. strain plots under uniaxial compression displayed a marked enhancement in the ultimate stress values, indicating the formation of tough double network hydrogels in comparison to their single network counterparts (Fig. 3B). In addition, the presence of a second network resulted in a significant increase in the compressive modulus from $8.13 \mathrm{kPa}$ to $17.09 \mathrm{kPa}$ (Fig. 3C and D). In the next step, we monitored the response of the single and double network systems under shear forces. An increase in yield stress as well as yield strain was detected from the stress and strain sweep plots (Fig. 3E). The ionically crosslinked alginate network
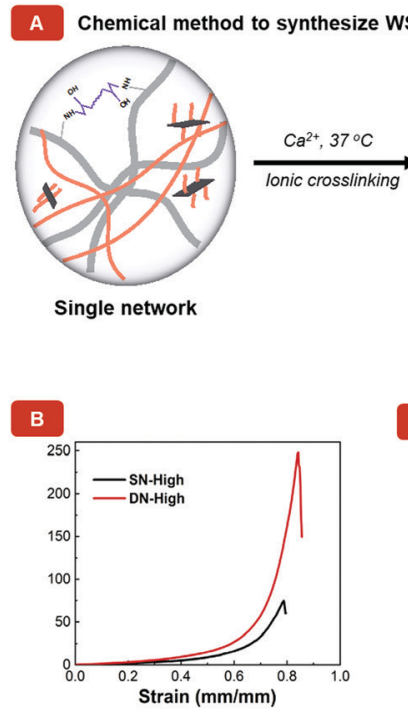

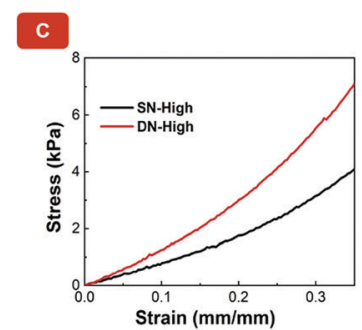

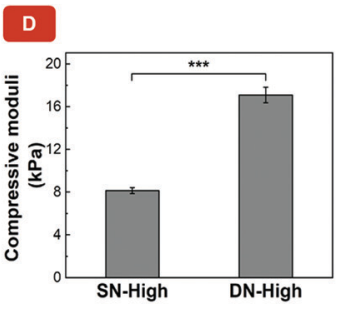

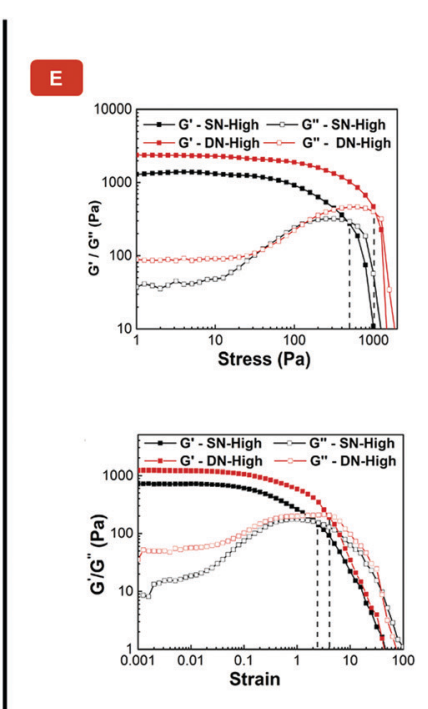

Fig. 3 Method for developing double network DNA-WS 2 hydrogels and comparing their mechanical properties with single-network counterparts. (A) Illustration displaying the chemical strategy for designing a double network nanocomposite hydrogel. Divalent calcium chloride ions crosslinked the carboxyl group of alginates to form the double network hydrogel. The image shows the appearance of this stable, double network hydrogel (scale bar $=$ $4 \mathrm{~mm}$ ). (B) Stress vs. strain plots of single and double network hydrogels containing the highest concentration of WS 2 . (C) Stress vs. strain plots magnified in the section from 0 to $0.35 \mathrm{~mm} \mathrm{~mm}^{-1}$ strain. (D) Comparison of the compressive moduli of the single and double network hydrogels. Results are represented as mean \pm standard deviation $(n=4)\left({ }^{* *} p<0.001\right)$. (E) Top panel: Stress-sweep comparison between single and double network systems. The dotted line represents the yield stress values for each gel. Bottom panel: Strain-sweep plots for the single and double network systems. The dotted line represents the yield strain values for each gel. 
enables the double network system to possess improved shear properties in comparison to the single network hydrogel.

\subsection{Physical characterization of $\mathrm{WS}_{2}$-Alg-impregnated single and double network hydrogels}

The surface morphology of the formulated hydrogels was monitored by scanning electron microscopy (SEM) (Fig. 4A). The single network hydrogels consisting of a distribution of $\mathrm{WS}_{2}$-Alg nanosheets in a DNA-based network displayed a porous morphology. Such a porous architecture may allow larger uptake of water, greater drug loading, and enhanced cellular infiltration and modulation. ${ }^{50}$ Additionally, the SEM micrograph of the double network hydrogel shows the maintenance of the porous structure even after the incorporation of a second network. The porosity of this nanocomposite material will allow for cell infiltration and nutrient transfer when used for tissue healing applications. In the next step the water uptake ability in the form of swelling behavior of the hydrogels was monitored. As expected, the swelling ratios of the double network hydrogels were lower compared to the single network nanocomposites over the entire timeframe of the study (Fig. 4B). The double network hydrogel consists of a tightly

\section{A}

C

Hydrogel placed inside the insert of transwell

crosslinked single network
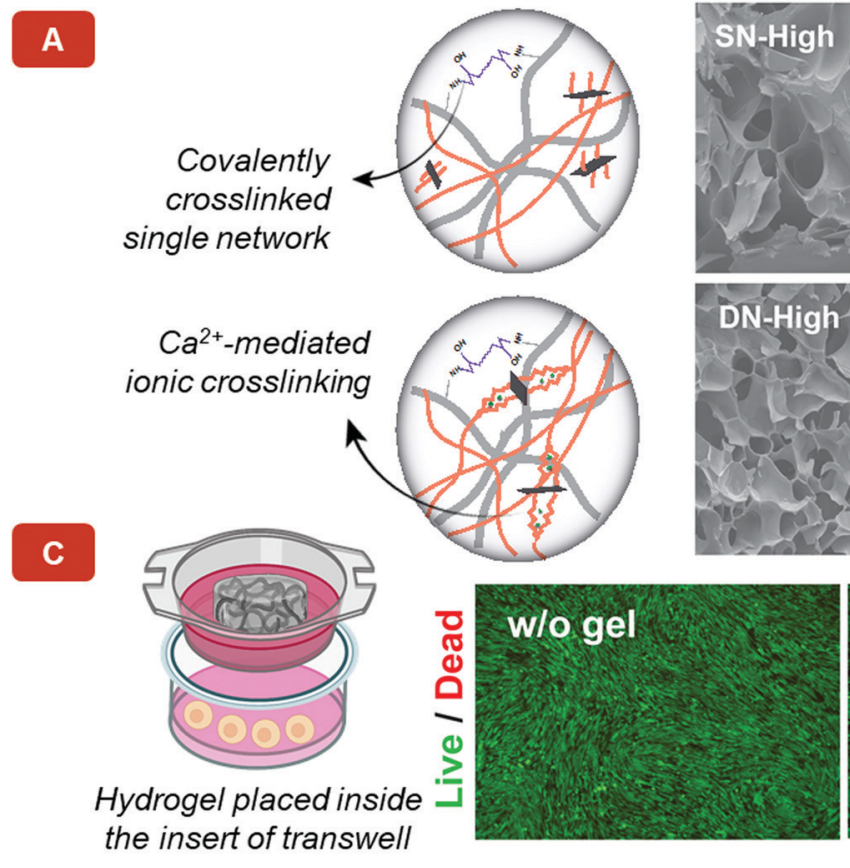
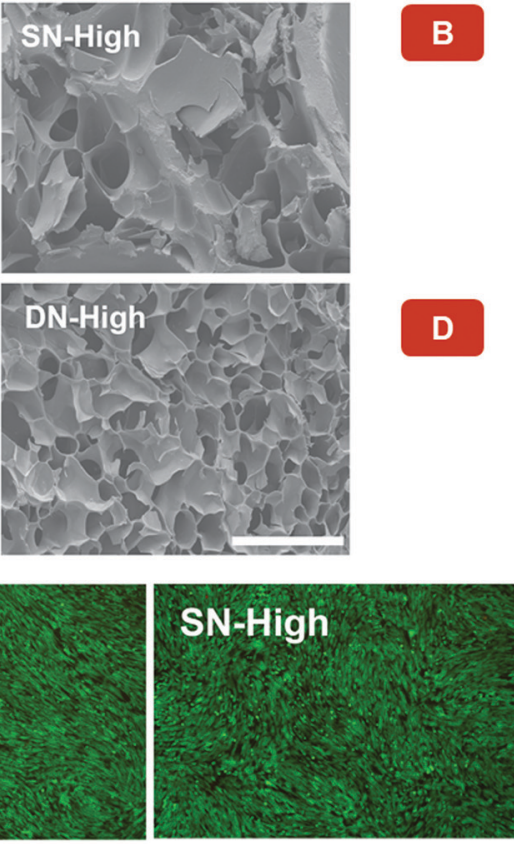

\section{B}

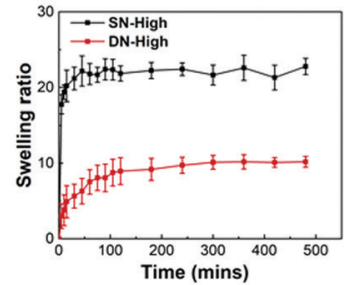

D
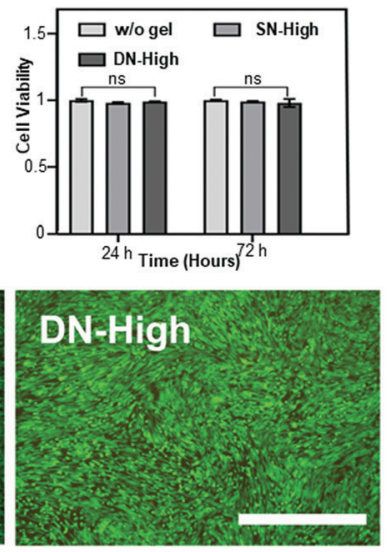

E

\section{DNA- WS2 Hydrogel properties}

- Can be prepared as Single Network and Double Network Polymeric Hydrogel

- Mechanically Resilient

- Porous with tunable mesh size

Biocompatible with Stem Cells

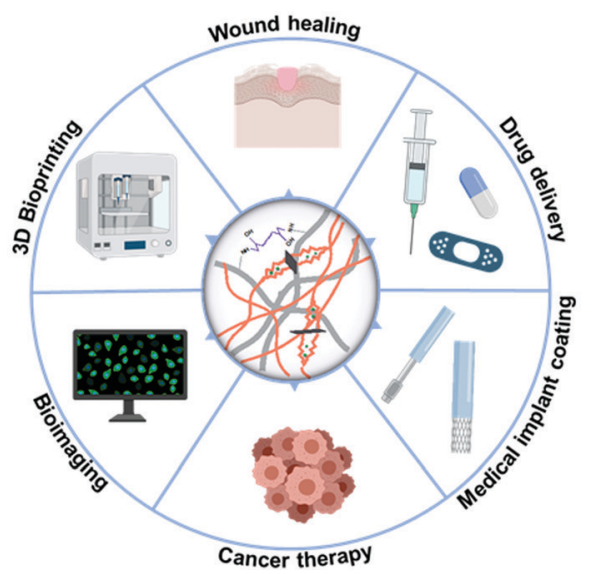

Fig. 4 Physical characterization of DNA-WS 2 hydrogels, evaluation of their in vitro biocompatibility, and proposing their applications. (A) Scanning electron micrographs showing the porous polymeric mesh of single and double network hydrogels containing the "high" concentration of exfoliated $W_{2}$ (scale bar $=200 \mu \mathrm{m}$ ). (B) Comparison of the swelling ratios for single and double network hydrogels as a function of time. Results are shown as mean \pm standard deviation $(n=4)$. (C) Fluorescence micrographs depicting the biocompatibility of the designed nanocomposite hydrogels. Human adipose-derived stem cells were exposed to the designed hydrogels for 72 hours, after which the fluorescence images were collected (scale bar $=1000 \mu \mathrm{m}$ ). (D) MTS assay of human adipose stem cells after 24 and 72 hours of contact with the respective hydrogels. Results are shown as mean \pm standard deviation ( $n=3$ ). (E) Table describing the relevant features of the designed DNA-WS 2 hydrogels. These hydrogels can be used for diverse biomedical applications as proposed in the illustration. 
crosslinked structure with a combination of two networks and a higher crosslink density. The higher extent of entanglements or crosslinks is responsible for the detected decrease in swelling ratios. The observation of reduced swelling ratios is consistent with earlier studies with double network hydrogels. ${ }^{51}$

\subsection{Evaluation of in vitro biocompatibility of the formulated nanocomposite hydrogels}

Finally, to envision the application of the designed nanocomposite hydrogels in tissue engineering, it was necessary to evaluate their biocompatibility. The in vitro biocompatibility was assessed by performing two cytotoxicity assays with human adipose stem cells (hASCs). Such stem cells were selected for the assays as it is easy to harvest them with high proliferation rates. ${ }^{52}$ Furthermore, the cytotoxicity assays were conducted by placing the gels inside cell culture inserts. This non-contact method of testing the cytotoxicity of hydrogels avoids cell apoptosis in adherent cell cultures arising from weightinduced mechanical stress. ${ }^{53}$ The stem cells were visualized via live-dead staining assays after growing them in contact with the formulated hydrogels. The cells for both the groups of single and double network hydrogels were well spread with a healthy morphology similar to the ones without any gel (Fig. 4C). Next, MTS assays, performed after 24 and 72 hours, displayed greater than $95 \%$ cell viability for both single and double network systems (Fig. 4D). Furthermore, nonsignificant differences in cell viability were observed after the incorporation of the second network. These results confirm the non-toxic nature of $\mathrm{WS}_{2}$ as well as the formulated single and double network hydrogels. The components of the hydrogel did not elicit any cytotoxic effects, thereby maintaining the cell morphology and viability. Previous reports have also shown the biocompatibility of $\mathrm{WS}_{2}$ nanosheets. ${ }^{16,54}$ Lastly, Fig. 4E highlights the critical features of the designed nanocomposite scaffolds. Most notably, the developed hydrogels are mechanically robust, porous, and biocompatible, which may lead to drug delivery and tissue engineering-based applications. Based on the applicability of similar nanocomposite hydrogel-based scaffolds, ${ }^{55-58}$ we also propose different possible biomedical applications of our nanocomposite DNA based hydrogel. However, in our study, we did not encapsulate the cells within the hydrogel or seeded the cells on top of it. For a direct-contact approach, the current formulation would require surface functionalization that incorporates cell adhesive motifs. Instead, a noncontact 2D cell culture model was used to represent the hydrogel as a drug delivery depot. Such non-contact 2D representation has its own limitations: ${ }^{59}$ (a) it does not accurately represent the $3 \mathrm{D}$ environment of the native tissue, and (b) the cell-cell interaction is different from the native tissue. Surface functionalization of these hydrogels with extracellular matrix-derived ligands is subject to future research when it is aimed to be used as a $3 \mathrm{D}$ tissuemimicking scaffold. Therefore, the developed nanocomposite DNA based hydrogel formulation has to go through further rigorous biological characterization, at cellular and molecular levels, before it can be adopted as a new healthcare material for diverse biomedical applications.

\section{Conclusions}

In summary, this study demonstrates the development of a double network nanocomposite hydrogel containing ultrathin, 2D nanosheets of a transition metal dichalcogenide (TMD). We have explored the potential of $\mathrm{WS}_{2}$ to form hydrogel scaffolds using DNA as the base polymer matrix. The nanocomposite scaffold exhibited an increase in compressive and shear strength in contrast to the DNA-only formulation. Additionally, the DNA-WS $\mathrm{W}_{2}$ based network was reinforced with an ionically crosslinked alginate network, resulting in the formation of a double network hydrogel. The double network hydrogels displayed a marked increase in compressive moduli, yield stress, and yield strains compared to their single network counterparts. The porous morphology of the DNA-based network was maintained after the addition of the second network. The hydrogel formulations were biocompatible since they did not elicit any cytotoxic effects or hinder cell growth and proliferation when human adipose stem cells were grown in contact with them. Therefore, stem cells can be delivered using these scaffolds, and they can potentially be used for tissue engineering applications. With further studies, this new hydrogel platform can also be used for the delivery of therapeutic molecules.

\section{Conflicts of interest}

The authors declare no conflicts of interest.

\section{Acknowledgements}

A. P. is grateful for the funding and support from the Canada Research Chairs Program of the Natural Sciences and Engineering Research Council (NSERC) of Canada, NSERC Discovery Grant, NSERC Discovery Accelerator Supplements (DAS), New Frontiers in Research Fund (NFRF)-Exploration Stream, Early Research Award (ERA) from the Province of Ontario, The Center for Advanced Materials and Biomaterials Research (CAMBR) Seed Grant, Western Strategic Support-CIHR Seed Grant, and WolfeWestern Fellowship At-Large for Outstanding Newly Recruited Research Scholar. Y. S. would like to acknowledge the funding and support from the Ontario Graduate Scholarship (OGS). The authors would also like to acknowledge Mr. Reza Khazaee and the Biotron facility at the University of Western Ontario for the transmission electron microscopy images. The authors are also thankful to the company, Biorender. Some illustrations were prepared with Biorender.com.

\section{References}

1 J. K. Carrow, K. A. Singh, M. K. Jaiswal, A. Ramirez, G. Lokhande, A. T. Yeh, T. R. Sarkar, I. Singh and A. K. Gaharwar, Proc. Natl. Acad. Sci. U. S. A., 2020, 117, 13329-13338.

2 C. Tan and H. Zhang, Chem. Soc. Rev., 2015, 44, 2713-2731.

3 L. Zong, X. Li, X. Han, L. Lv, M. Li, J. You, X. Wu and C. Li, ACS Appl. Mater. Interfaces, 2017, 9, 32280-32289. 
4 T. Liu, C. Wang, X. Gu, H. Gong, L. Cheng, X. Shi, L. Feng, B. Sun and Z. Liu, Adv. Mater., 2014, 26, 3433-3440.

5 T. I. Kim, B. Kwon, J. Yoon, I. J. Park, G. S. Bang, Y. K. Park, Y. S. Seo and S. Y. Choi, ACS Appl. Mater. Interfaces, 2017, 9, 7908-7917.

6 V. Agarwal and K. Chatterjee, Nanoscale, 2018, 10, 16365-16397.

7 Y. Chen, C. Tan, H. Zhang and L. Wang, Chem. Soc. Rev., 2015, 44, 2681-2701.

8 H. P. Lee and A. K. Gaharwar, Adv. Sci., 2020, 7, 2000863.

9 V. Urbanová and M. Pumera, Nanoscale, 2019, 11, 15770-15782.

10 L. Gong, L. Yan, R. Zhou, J. Xie, W. Wu and Z. Gu, J. Mater. Chem. B, 2017, 5, 1873-1895.

11 R. Zhou, S. Zhu, L. Gong, Y. Fu, Z. Gu and Y. Zhao, J. Mater. Chem. B, 2019, 7, 2588-2607.

12 S. Anju and P. V. Mohanan, Synth. Met., 2021, 271, 116610.

13 D. Xuan, Y. Zhou, W. Nie and P. Chen, Carbohydr. Polym., 2017, 155, 40-48.

14 G. Guan, S. Zhang, S. Liu, Y. Cai, M. Low, C. P. Teng, I. Y. Phang, Y. Cheng, K. L. Duei, B. M. Srinivasan, Y. Zheng, Y. W. Zhang and M. Y. Han, J. Am. Chem. Soc., 2015, 137, 6152-6155.

15 G. Guan, J. Xia, S. Liu, Y. Cheng, S. Bai, S. Y. Tee, Y.-W. Zhang and M.-Y. Han, Adv. Mater., 2017, 29, 1700326.

16 G. S. Bang, S. Cho, N. Son, G. W. Shim, B. K. Cho and S. Y. Choi, ACS Appl. Mater. Interfaces, 2016, 8, 1943-1950.

17 S. Wang, J. Zhao, H. Yang, C. Wu, F. Hu, H. Chang, G. Li, D. Ma, D. Zou and M. Huang, Acta Biomater., 2017, 58, 442-454.

18 Z. Lei, W. Zhu, S. Sun and P. Wu, Nanoscale, 2016, 8, 18800-18807.

19 J. E. Kim, D. Yim, S. W. Han, J. Nam, J. H. Kim and J. W. Kim, ACS Appl. Mater. Interfaces, 2019, 11, 18817-18824.

20 A. S. Hoffman, Adv. Drug Delivery Rev., 2012, 64, 18-23.

21 K. Y. Lee and D. J. Mooney, Chem. Rev., 2001, 101, 1869-1879.

22 J. Li and D. J. Mooney, Nat. Rev. Mater., 2016, 1, 16071.

23 A. K. Gaharwar, I. Singh and A. Khademhosseini, Nat. Rev. Mater., 2020, 5, 686-705.

24 S. Polla Ravi, Y. Shamiya, A. Chakraborty, C. Elias and A. Paul, Trends Pharmacol. Sci., 2021, 42, 813-828.

25 H. Uludag, A. Pandit and L. Kuhn, Front. Bioeng. Biotechnol., 2020, 0, 559.

26 Z. Liu, Y. Faraj, X.-J. Ju, W. Wang, R. Xie and L.-Y. Chu, J. Polym. Sci., Part B: Polym. Phys., 2018, 56, 1306-1313.

27 A. K. Gaharwar, N. A. Peppas and A. Khademhosseini, Biotechnol. Bioeng., 2014, 111, 441-453.

28 S. Merino, C. Martín, K. Kostarelos, M. Prato and E. Vázquez, ACS Nano, 2015, 9, 4686-4697.

29 S. Pacelli, R. Maloney, A. R. Chakravarti, J. Whitlow, S. Basu, S. Modaresi, S. Gehrke and A. Paul, Sci. Rep., 2017, 7, 1-15.

30 A. Chakraborty, A. Roy, S. Polla Ravi and A. Paul, Biomater. Sci., 2021, 9, 6337-6354.

31 Z. Zohreband, M. Adeli and A. Zebardasti, Int. J. Biol. Macromol., 2021, 182, 2048-2055.

32 J. Gačanin, C. V. Synatschke and T. Weil, Adv. Funct. Mater., 2020, 30, 1906253.

33 F. Li, D. Lyu, S. Liu and W. Guo, Adv. Mater., 2020, 32, 1806538.
34 S. Basu, S. Pacelli, Y. Feng, Q. Lu, J. Wang and A. Paul, ACS Nano, 2018, 12(10), 9866-9880.

35 S. Basu, R. Johl, S. Pacelli, S. Gehrke and A. Paul, ACS Macro Lett., 2020, 9, 1230-1236.

36 A. Chakraborty, S. P. Ravi, Y. Shamiya, C. Cui and A. Paul, Chem. Soc. Rev., 2021, 50, 7779-7819.

37 S. Basu, A. R. Alkiswani, S. Pacelli and A. Paul, ACS Appl. Mater. Interfaces, 2019, 11, 34621-34633.

38 Y. Xu, Q. Wu, Y. Sun, H. Bai and G. Shi, ACS Nano, 2010, 4, 7358-7362.

39 P. K. Pandey, H. Ulla, M. N. Satyanarayan, K. Rawat, A. Gaur, S. Gawali, P. A. Hassan and H. B. Bohidar, ACS Appl. Nano Mater., 2020, 3, 1289-1297.

40 M. A. Haque, T. Kurokawa and J. P. Gong, Polymer, 2012, 53, 1805-1822.

41 J. P. Gong, Soft Matter, 2010, 6, 2583-2590.

42 M. Sahu, L. Narashimhan, O. Prakash and A. M. Raichur, ACS Appl. Mater. Interfaces, 2017, 9, 14347-14357.

43 C. Zhang, D. F. Hu, J. W. Xu, M. Q. Ma, H. Xing, K. Yao, J. Ji and Z. K. Xu, ACS Nano, 2018, 12, 12347-12356.

44 G. Lalwani, A. M. Henslee, B. Farshid, P. Parmar, L. Lin, Y. X. Qin, F. K. Kasper, A. G. Mikos and B. Sitharaman, Acta Biomater., 2013, 9, 8365-8373.

45 M. Naffakh, C. Marco and G. Ellis, CrystEngComm, 2014, 16, 5062-5072.

46 F. Yin, T. Anderson, N. Panwar, K. Zhang, S. C. Tjin, B. K. Ng, H. S. Yoon, J. Qu and K. T. Yong, Nanotheranostics, 2018, 2, 371-386.

47 C. Lu, Y. Liu, Y. Ying and J. Liu, Langmuir, 2017, 33, 630-637.

48 H. Vovusha and B. Sanyal, RSC Adv., 2015, 5, 67427-67434.

49 K. Y. Lee and D. J. Mooney, Prog. Polym. Sci., 2012, 37, 106-126.

50 S. Pacelli, A. R. Chakravarti, S. Modaresi, S. Subham, K. Burkey, C. Kurlbaum, M. Fang, C. A. Neal, A. J. Mellott, A. Chakraborty and A. Paul, J. Biomed. Mater. Res., Part A, 2021, 109(12), 2597-2610.

51 Q. Chen, H. Chen, L. Zhu and J. Zheng, J. Mater. Chem. B, 2015, 3, 3654-3676.

52 S. Pacelli, S. Basu, C. Berkland, J. Wang and A. Paul, Cell. Mol. Bioeng., 2018, 11, 211-217.

53 M. Kempf, R. M. Kimble and L. Cuttle, Burns, 2011, 37, 994-1000.

54 J. H. Appel, D. O. Li, J. D. Podlevsky, A. Debnath, A. A. Green, Q. H. Wang and J. Chae, ACS Biomater. Sci. Eng., 2016, 2, 361-367.

55 M. K. Jaiswal, J. K. Carrow, J. L. Gentry, J. Gupta, N. Altangerel, M. Scully and A. K. Gaharwar, Adv. Mater., 2017, 29, 1702037.

56 Q. Xu, S. Zhao, L. Deng, J. Ouyang, M. Wen, K. Zeng, W. Chen, L. Zhang and Y. N. Liu, Chem. Commun., 2019, 55, 9471-9474.

57 K. Kasinathan, K. Marimuthu, B. Murugesan, S. Samayanan, Y. Cai and C. Rathinam, J. Mol. Liq., 2021, 335, 116582.

58 J. Azadmanjiri, P. Kumar, V. K. Srivastava and Z. Sofer, ACS Appl. Nano Mater., 2020, 3, 3116-3143.

59 C. Jensen and Y. Teng, Front. Mol. Biosci., 2020, 7, 33. 